\title{
The impact of Frailty on complications in patients undergoing common urologic procedures; a study from the American College of Surgeons National Surgical Quality Improvement Database
}

\author{
Anne M Suskind ${ }^{1}$, Louise C Walter ${ }^{2}$, Chengshi Jin², John Boscardin ${ }^{3}$, Saunak Sen ${ }^{3}$, \\ Matthew R Cooperberg ${ }^{1}$, and Emily Finlayson ${ }^{2,4}$ \\ ${ }^{1}$ University of California, San Francisco Department of Urology \\ ${ }^{2}$ University of California, San Francisco and San Francisco VA Medical Center Division of \\ Geriatrics \\ ${ }^{3}$ Universtiy of California, San Francisco Department of Epidemiology and Biostatistics \\ ${ }^{4}$ University of California, San Francisco Department of Surgery, Division of General Surgery
}

\begin{abstract}
Objectives-To evaluate the association of frailty, a measure of diminished physiologic reserve, with both major and minor surgical complications among patients undergoing urologic surgery.

Materials and Methods-Using data from the American College of Surgeons National Surgical Quality Improvement Program (ACS-NSQIP) from 2007 to 2013, we identified all urologic cases that appeared more than 1000 times in the dataset among patients age 40 and older. Frailty was measured using the NSQIP Frailly Index (FI), a validated measure that includes 11 impairments such as decreased functional status and impaired sensorium. We created multivariable logistic regression models using the NSQIP Frailty Index to assess major and minor complications after surgery.
\end{abstract}

Results-We identified 95,108 urologic cases representing 21 urologic procedures. The average frequency of complications per individual was $11.7 \%$, with the most common complications being hospital readmission (6.2\%), blood transfusion (4.6\%), and urinary tract infection (3.1\%). Major and minor complications increased with increasing NSQIP-FI. Frailly remained strongly associated with complications after adjustment for year, age, race, smoking status, and method of anesthesia [adjusted OR 1.74 (95\% CI 1.64, 1.85) NSQIP-FI 0.18+]. Increasing NSQIP-FI was associated with increasing frequency of complications within age groups (by decade) up to age 81 and across most procedures.

Corresponding Author: Anne M. Suskind, MD, MS, 400 Parnassus Avenue, Box 0738, San Francisco, CA 94143-0738, (415)476-1611, Anne.Suskind@ucsf.edu.

The American College of Surgeons National Surgical Quality Improvement Program and the hospitals participating in the ACS NSQIP are the source of the data used herein; they have not verified and are not responsible for the statistical validity of the data analysis or the conclusions derived by the authors.

Conflicts of Interest: None disclosed 
Conclusion-Frailty strongly correlates with risk of post-operative complications among patients undergoing urologic surgery. This finding is true within most age groups and across most urologic procedures.

\section{Keywords}

meta-analysis; general urology age; disability

\section{Introduction}

Approximately two thirds of all urologic procedures are performed in patients ages 65 and older.(1) As this population continues to grow at a rapid rate - nearly one in five United States residents will be age 65 and older by the year 2030(2) - urologists will be increasingly confronted with the complexities of surgical decision making and care in older individuals.

Among these complexities is the presence of frailty. Frailty is a measure of physiological vulnerability that often manifests in older individuals as an increased susceptibility to disability. $(3,4)$ Frailty results from cumulative declines across multiple organ systems that often leads to deterioration and adverse events in response to stressors, such as surgery.(5) This concept distinguishes frailty from potential causes such as comorbidity and from potential downstream outcomes such as disability. Frailty, which is often treated as synonymous with comorbidity, is in fact a distinct concept represented by an accumulation of conditions in combination with a decline in function. Frailly has been linked to poor postoperative outcomes in older individuals undergoing general surgical(6-10) and urologic oncology(11) procedures, including increased complications, prolonged length of stay and discharge to a skilled or assisted living facility. The effect of frailty on the majority of urologic procedures commonly performed in older adults, however, has yet to be determined.

Using data from the American College of Surgeons National Surgical Quality Improvement Program (ACS NSQIP), we examined the association between frailty and both major and minor complications among patients undergoing common urologic procedures in the United States from 2007 to 2013. Findings from this study will help urologists to better target surgical procedures for patients in whom the benefits of surgery will outweigh the risks.

\section{Materials and Methods}

\section{Patients and Databases}

We used data from the American College of Surgeons National Quality Improvement Project (ACS NSQIP) Participant Data Use File from 2007 to 2013. This study was determined to be exempt by our institution's Institutional Review Board. The NSQIP database compiles data from hospitals that voluntarily contribute information pertaining to their sample of surgical procedures. Highly trained surgical clinical reviewers who are employed by the hospital and trained by ACS examine medical records and classify perioperative clinical data 
and outcomes up to 30 days. As of 2014, over 445 hospitals nationwide contributed cases to this database.(12)

From this population, we identified all urologic cases using the surgical specially variable. Since many sling procedures are performed by both urologists and gynecologists, this procedure was searched for by Current Procedure Terminology (CPT) code 57288 [sling operation for stress incontinence (eg, fascia or synthetic)] and all sling procedures were included, regardless of specially type. We focused on commonly performed proceduresprocedures that had over 1,000 entries in the database - which ensured adequate sample size. In order to include a breadth of cases, no exclusionary criteria were applied to allow for the generalizability of our findings.

\section{Outcomes}

Thirty day complications were ascertained from the NSQIP database. Complications were classified as either minor [eg, urinary tract infection (UTI), superficial wound infection, pneumonia and blood transfusion] or major [eg, hospital readmission, return to the operating room, sepsis, deep vein thrombosis (DVT), cerebral vascular accident (CVA), reintubation, renal failure, myocardial infarction, pulmonary embolus, septic shock, dehiscence, cardiac arrest, deep wound infection, coma and death] in accordance with prior studies looking at complications using NSQIP data. $(13,14)$

\section{Covariates}

Frailly was assessed using a frailly index (FI) designed specifically for use in NSQIP data. This index was adapted from the Canadian Study of Health and Aging-frailty index (CSHAFI) which includes factors that can be easily elicited from the patient history and have been determine to have greater precision for measuring frailty than the Charlson Comorbidity index. The NSQIP-FI includes 11 items: (1) history of diabetes, (2) impaired functional status, (3) history of chronic obstructive pulmonary disease (COPD) or pneumonia, (4) history of congestive heart failure, (5) history of myocardial infarction within 6 months of surgery, (6) history of percutaneous coronary intervention, cardiac surgery, or angina, (7) being on hypertensive medications, (8) peripheral vascular disease or rest pain, (9) impaired sensorium, (10) transient ischemic attack or cerebral vascular accident defined as focal neurologic deficits of sudden onset and brief duration, and (11) cerebrovascular accident with deficit defined by history of cerebrovascular accident with persistent residual dysfunction. Items are summed and divided by 11 for the composite FI score, so that a NSQIP FI score of $1 / 11$ would be 0.09 , a NSQIP FI score of $2 / 11$ would be 0.18 , and so on and so forth. This index has been shown to demonstrate excellent correlation with both mortality and morbidity in all surgical subspecialties, including urology, and distinctly measures frailty, as opposed to comorbidity.(15)

Additional covariates including demographic [age, race, smoking status, recent weight loss (defined as $>10 \%$ loss of body weight in the last 6 months), serum creatinine, albumin and hematocrit] and case-based characteristics (year of case, and type of anesthesia) were obtained from the NSQIP files. 


\section{Statistical Analysis}

Demographic and case-based characteristics of patients undergoing urologic procedures were reported as frequencies and percentages. Unadjusted and adjusted logistic regression models were used to explore which factors were associated with complications resulting from urologic procedures. Models were adjusted for year, age, race, type of anesthesia, smoking status, and NSQIP FI. Other variables (e.g., recent weight loss, serum creatinine, albumin, and hematocrit) were not included in the models due to potential overlap with the NSQIP FI.

In order to determine the effect of frailty on complications for individual urologic procedures, we used a two-level random effects model in which the association between frailty and complications is assessed within each procedure, and then across procedures.

More specifically, since each procedure has a unique clinical context, we performed separate multivariable logistic regression analyses for each procedure, assessing the association between frailty and complications. The models were adjusted for year, age, race, type of anesthesia and smoking status. The log odds ratios (and 95\% CI's) were combined in using a random effects meta analysis approach to provide an overall picture of the association between frailty and complications across procedures. Heterogeneity across procedures was assessed using an $\mathrm{I}^{2}$ statistic.

\section{Results}

We identified 95,108 cases representing 21 different urologic procedures in the NSQIP database from 2007-2013. Demographic and case based characteristics are described in Table 1. The majority (67.8\%) of urologic procedures were performed in individuals ages 61 and older. Additionally, the majority of urologic patients were white $(89.4 \%)$, non-smokers (84.7\%), and did not have recent weight loss (99.1\%). Almost $40 \%$ of patients had no frailly items, $38.9 \%$ had 1 frailty item and $21.6 \%$ had 2 or more frailly items. More cases were available as the years advanced, with over thirty percent of the cases being done in 2013. The majority of cases were performed using general anesthesia (89\%).

Figure 1 demonstrates complications for each of the 21 procedures stratified into major complications, minor complications, and a combination of major and minor complications. Cystectomy with lymph node dissection had the highest percentage of complications at $58.2 \%$, while hydrocele had the lowest percentage of complications at $5.4 \%$.

Frequencies of individual complications are depicted in Table 2. The overall complication rate was $11.7 \%$. The most common minor complication was blood transfusion $(4.6 \%)$ followed by urinary tract infection $(3.1 \%)$. The most common major complication was hospital readmission $(6.2 \%)$.

Results of the logistic regression predicting factors associated with complications are shown in Table 3. Increasing frailty was significantly associated with increasing odds of complications in both unadjusted and adjusted models; for NSQIP FI=0.09 adjusted OR 1.28 (95\% CI 1.21, 1.36) and for NSQIP FI=0.18 and greater adjusted OR 1.74 (95\% CI 1.21, 
1.36). Increasing age was also associated with increased odds of complications by decade in both models; ages 51-60 adjusted OR 1.21 (95\% CI 1.10, 1.34), ages 61-70 adjusted OR 1.27 (95\% CI 1.16, 1.39), ages 71-80 adjusted OR 1.61 (95\% CI 1.46, 1.77), ages 81 plus adjusted OR 1.99 (95\% CI 1.79, 2.20). Patients who smoked had increased odds of having complications compared to those who did not smoke in the adjusted model with an adjusted OR of 1.16 (95\% CI 1.09, 1.23). Cases performed with monitored anesthesia care (MAC) were associated with lower odds of complications compared to those performed under general anesthesia in the adjusted model, adjusted OR 0.55 (95\% CI 0.46, 0.67).

Additionally, cases performed in more recent calendar years, 2011 to 2013 in the adjusted model, had higher odds of having complications compared to procedures performed in the year 2007. Of note, we did perform a separate model to evaluate for possible interaction between frailty and age. We found that this interaction was not significant with an overall $\mathrm{p}$ value of 0.29 (results not shown).

Figure 2 further illustrates the association between the NSQIP FI and complications. As the NSQIP FI increases, rates of complications also increase. This holds true for complications overall and for major and minor complications individually. Figure 3 combines age (in decades) and NSQIP FI score to provide the percentage of overall complications with $95 \%$ confidence intervals (represented by error bars) for each age-NSQIP FI group NSQIP FI was significantly associated with increased complications within age groups. For example, in the 40 to 50 age group, individuals with a NSQIP FI of 0 had a significantly lower percentage of complications compared to individuals of the same decade of age with a NSQIP FI of $0.18+$. This trend holds true for all age groups up until the 80+ age group, which demonstrates similar percentages of complications across different NSQIP FI categories. Findings were similar for both major and minor complications (data not shown).

We found that frailty was associated with an increased risk of complications in nearly all urologic procedures that we studied (with $\mathrm{p}<0.01$ ), as shown in Figure 4. Exceptions included prostatectomy with lymph node dissection, prostatectomy with extended lymph node dissection, cystectomy, sling procedures for urinary incontinence and orchiectomy. Across procedures, frailty was associated with an increased risk of complications with a log OR of 0.237 (95\% CI 0.183, 0.2901). However, we also observed substantial heterogeneity across procedures $\left(\mathrm{I}^{2}=55.9 \%, \mathrm{p}<0.01\right)$.

\section{Discussion}

We found that frailty is strongly associated with both major and minor complications among patients undergoing urologic surgery. This finding was consistent across and within age groups up to age 81 , where complications were similar despite frailty status. Additionally, frailty had a significant association with complications for most urologic procedures examined. Other factors associated with complications included increasing age, smoking status, cases performed under general anesthesia, and more recent calendar year, likely due to increased case mix and complexity with the maturation of the NSQIP database.

Our findings are consistent with prior studies among patients undergoing non-urologic procedures that have found a significant association between frailty and complications. One 
study examined patients undergoing elective surgical procedures and found that preoperative frailty, defined by the Fried criteria (weakness, weight loss, exhaustion, low physical activity, and slowed walking speed), was associated with an increased risk for post-operative complications, length of hospital stay, and discharge to a skilled or assisted-living facility.(6) Studies performed in the vascular literature have demonstrated similar findings. Frail patients undergoing emergent and elective vascular procedures were found to have longer hospital stays and poorer postoperative outcomes, $(8,9)$ and an association between frailty and mortality has been demonstrated among patients undergoing transcatheter aortic valve replacement.(7) Another study looked at patients undergoing major intra-abdominal procedures and found that two specific components of frailty, shrinking and grip strength, were highly predictive of 30-day morbidity and mortality.(11)

Interestingly, our study demonstrated an association between frailty and complications independent of age, demonstrated by a p value of 0.29 for interaction between these two variables. We found that within each decade of life, with the exception of octogenarians, increasing frailty was significantly associated with higher percentages of complications. In other words, a frail 40-50 year old has a similar frequency of complications as an 81 year old and a higher frequency of complications than a non-frail 40-50 year old. This trend was similar for all decades of life up to age 81 and for both minor and major complications separately.

Our study is the first to evaluate frailty according to several individual urologic procedures that are commonly performed. We found that with the exception of the following procedures: cystectomy, prostatectomy with extended and non-extended lymph node dissection, sling procedures for stress urinary incontinence and orchiectomy; that increasing frailty was associated with increased complications across most urologic procedures. While these exceptions did not reach statistical significance, they were all very near the threshold of significance and interpretation of these results should be interpreted with this in mind. What is striking about the evaluation of the association between increasing frailty and complications within individual procedures, however, is that almost all procedures, both "major" (i.e. various partial and radical nephrectomy) and "minor" (i.e. transurethral resection of the prostate and hydrocele) demonstrated this significant trend $(\mathrm{p}<0.01)$, highlighting the importance of the evaluation of frailty among all individuals undergoing all types of urologic surgery.

This study should be considered with several limitations in mind. First, we are limited by the nature of the NSQIP database in the level of detail of the information that is available for analysis. Second, hospitals that participate in NSQIP may have certain resources and qualities that differentiate them from hospitals that do not participate, making findings from this study less generalizable to non-NSQIP affiliated hospitals. Additionally, due to the restraints of the data structure, we are only able to analyze complications within 30 days of the index procedures and are not able to capture any longer-term outcomes or complications. We are also somewhat limited in our measure of frailty. Ideally we would be able to complete functional and cognitive assessments in each patient undergoing surgery, however, these data are not available and instead we used an already established and validated functional score previously designed for and used in these data. Additionally, there may be 
other factors that may help to improve upon the NSQIP-ASA frailty index, such as ASA classification, $(11,16)$ however, we chose not to include these factors in our analyses because we chose to focus on data that was readily available to urologists in the preoperative clinical setting. Finally, we chose to combine complications into "major" and "minor" categories, as opposed to other classification schemes such as the Clavien Dindo system, for ease of interpretation and data presentation. While we recognize that there are many different ways to divide up complications, the differentiation between major and minor complications has been used in similar studies with NSQIP data that are published in the literature.(13, 14)

Frailly is strongly associated with short-term postoperative complications among patients undergoing most urologic procedures. This association is significant across age groups, type of complication (major and minor), and various procedure types. These findings highlight the importance of preoperative frailly assessment and how this assessment can enhance surgical decision-making among physicians, patients, and their families for optimized postoperative outcomes.

\section{Acknowledgments}

Funding: Dr. Suskind's time was supported by the National Institute of Diabetes and Digestive Kidney Diseases (grant number K12 DK83021-07). Dr. Walter's work on this project was supported by the National Institute on Aging at the National Institutes of Health (grant number K24AG041180). This work was also supported by the UCSF CTSI Scholars Program and the UCSF Claude D. Pepper Older Americans Independence Center.

\section{References}

1. Drach GW, Griebling TL. Geriatric urology. Journal of the American Geriatrics Society. 2003; 51(7 Suppl):S355-8. [PubMed: 12823668]

2. U.S. Census Bureau. The next four decades; The older population in the United States: 2010 to 2050 population estimates and projections 2010 September 24 2014. Available from: http:// www.google.com/url?sa=t\&rct=j\&q=\&esrc=s\&source=web\&cd=l\&ved=OCCAQFjAA\&url=http $\% 3 \mathrm{~A} \% 2 \mathrm{~F} \% 2 \mathrm{Fwww} . c e n s u s . g o v \% 2 \mathrm{Fprod} \% 2 \mathrm{~F} 2010 \mathrm{pubs}$ \%2Fp25-1138.pdf\&ei=jgojVLfrH8iIoQThuYJY\&usg=AFQjCNEjIpOJ0zJcUsCUrtLOgz5JkXUPw\&sig2=pf3rVN7uGHldOjTEkQSn8w\&bvm=bv.76180860,d.cGU

3. Buchner DM, Wagner EH. Preventing frail health. Clinics in geriatric medicine. 1992; 8(1):1-17. [PubMed: 1576567]

4. Fried LP, Tangen CM, Walston J, Newman AB, Hirsch C, Gottdiener J, et al. Frailty in older adults: evidence for a phenotype. The journals of gerontology Series A, Biological sciences and medical sciences. 2001; 56(3):M146-56.

5. Gazala S, Tul Y, Wagg A, Widder SL, Khadaroo RG, Acute C, et al. Quality of life and long-term outcomes of octo- and nonagenarians following acute care surgery: a cross sectional study. World journal of emergency surgery : WJES. 2013; 8(1):23. [PubMed: 23816269]

6. Makary MA, Segev DL, Pronovost PJ, Syin D, Bandeen-Roche K, Patel P, et al. Frailty as a predictor of surgical outcomes in older patients. Journal of the American College of Surgeons. 2010; 210(6):901-8. [PubMed: 20510798]

7. Green P, Arnold SV, Cohen DJ, Kirtane AJ, Kodali SK, Brown DL, et al. Relation of Frailly to Outcomes After Transcatheter Aortic Valve Replacement (from the PARTNER Trial). The American journal of cardiology. 2015

8. Partridge JS, Fuller M, Harari D, Taylor PR, Martin FC, Dhesi JK. Frailty and poor functional status are common in arterial vascular surgical patients and affect postoperative outcomes. Int J Surg. 2015; 18:57-63. [PubMed: 25907322] 
9. Ambler GK, Brooks DE, Al Zuhir N, Ali A, Gohel MS, Hayes PD, et al. Effect of frailty on shortand mid-term outcomes in vascular surgical patients. The British journal of surgery. 2015; 102(6): 638-45. [PubMed: 25764503]

10. Chen CC, Lin MT, Liang JT, Chen CM, Yen CJ, Huang GH. Pre-surgical Geriatric Syndromes, Frailly, and Risks for Postoperative Delirium in Older Patients Undergoing Gastrointestinal Surgery: Prevalence and Red Flags. Journal of gastrointestinal surgery : official journal of the Society for Surgery of the Alimentary Tract. 2015; 19(5):927-34. [PubMed: 25650167]

11. Revenig LM, Canter DJ, Kim S, Liu Y, Sweeney JF, Sarmiento JM, et al. Report of a Simplified Frailty Score Predictive of Short-Term Postoperative Morbidity and Mortality. Journal of the American College of Surgeons. 2015; 220(5):904-11 el. [PubMed: 25907870]

12. American College of Surgeons National Quality Improvement Program (ACS NSQIP). Semiannual Report. 2014

13. Etzioni DA, Wasif N, Dueck AC, Cima RR, Hohmann SF, Naessens JM, et al. Association of hospital participation in a surgical outcomes monitoring program with inpatient complications and mortality. JAMA : the journal of the American Medical Association. 2015; 313(5):505-11. [PubMed: 25647206]

14. Molina CS, Thakore RV, Blumer A, Obremskey WT, Sethi MK. Use of the National Surgical Quality Improvement Program in orthopaedic surgery. Clinical orthopaedics and related research. 2015; 473(5):1574-81. [PubMed: 24706043]

15. Velanovich V, Antoine H, Swartz A, Peters D, Rubinfeld I. Accumulating deficits model of frailty and postoperative mortality and morbidity: its application to a national database. The Journal of surgical research. 2013; 183(1):104-10. [PubMed: 23415494]

16. Lascano D, Pak JS, Kates M, Finkelstein JB, Silva M, Hagen E, et al. Validation of a frailty index in patients undergoing curative surgery for urologic malignancy and comparison with other risk stratification tools. Urologic oncology. 2015 

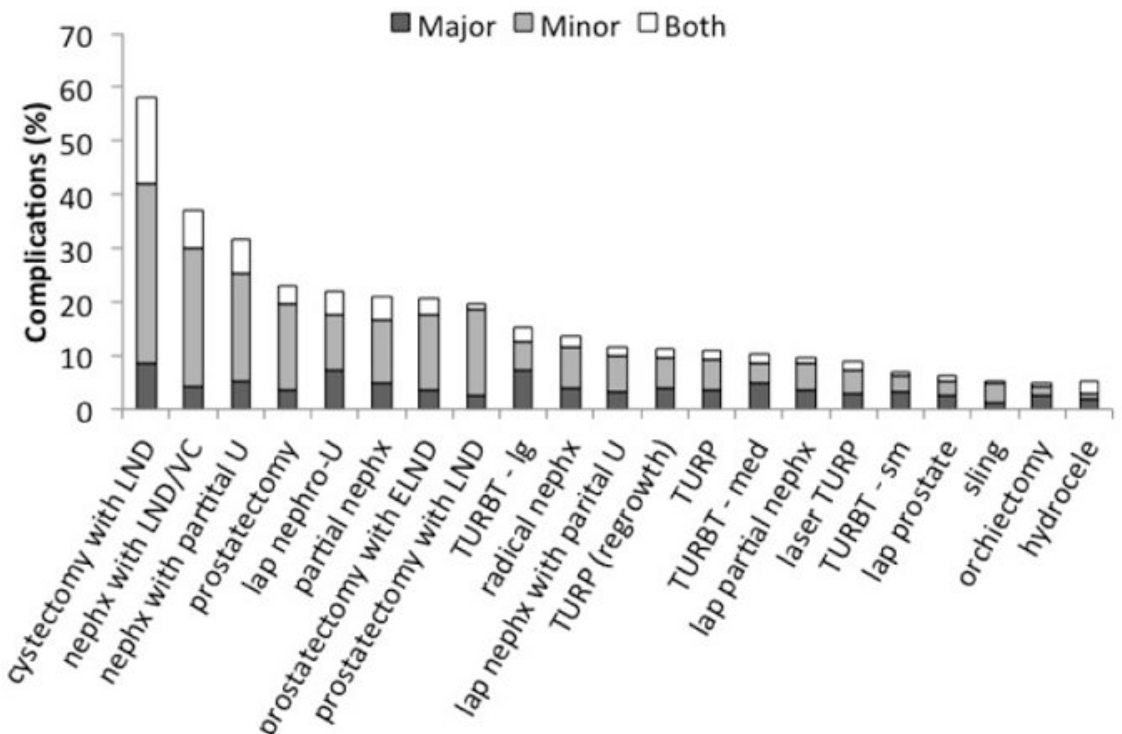

$\mathrm{LND}=$ lymph node dissection; $\mathrm{VC}=$ vena cava thrombectomy; $\mathrm{U}=$ ureterectomy; nephx=nephrectomy; ELND=extended LND; TURBT=transurethral resection of bladder tumor; Ig=large; lap=laparoscopic; TURP=transurethral resection of prostate; med=medium, sm=small

Figure 1. Frequency of complications of common urologic procedures 


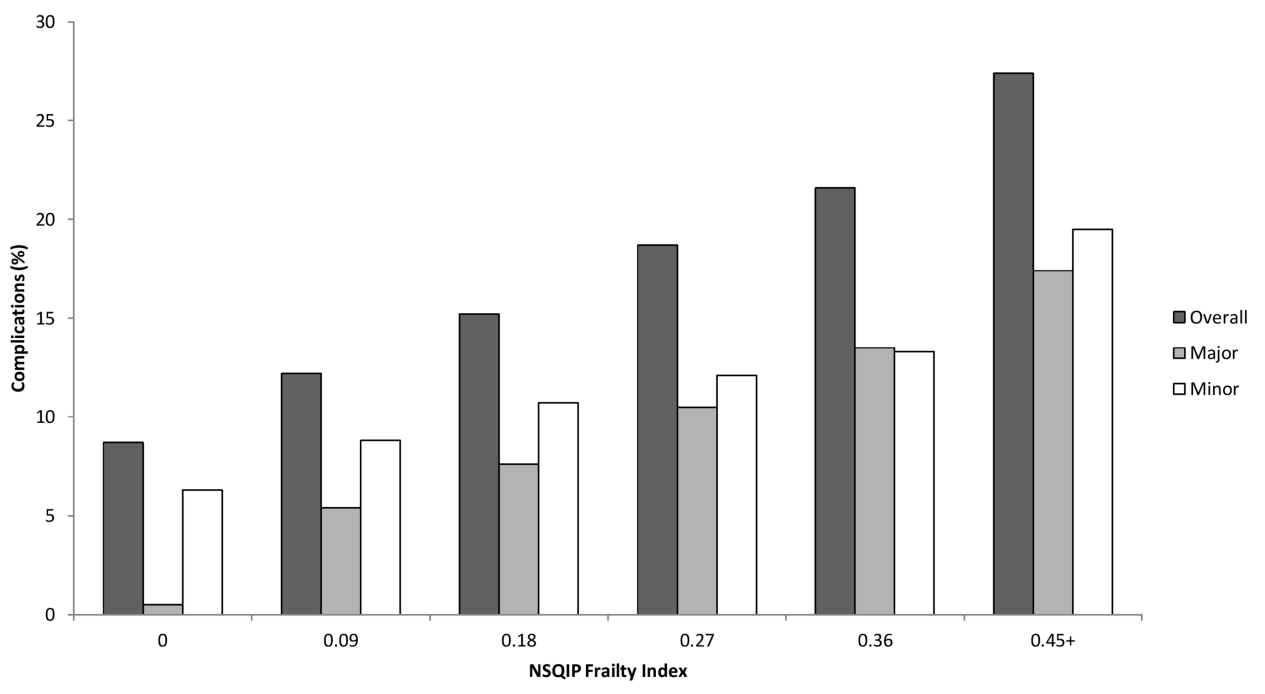

Figure 2. Frequency of urologic procedures complications by NSQIP Frailty Index

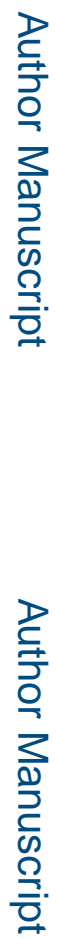




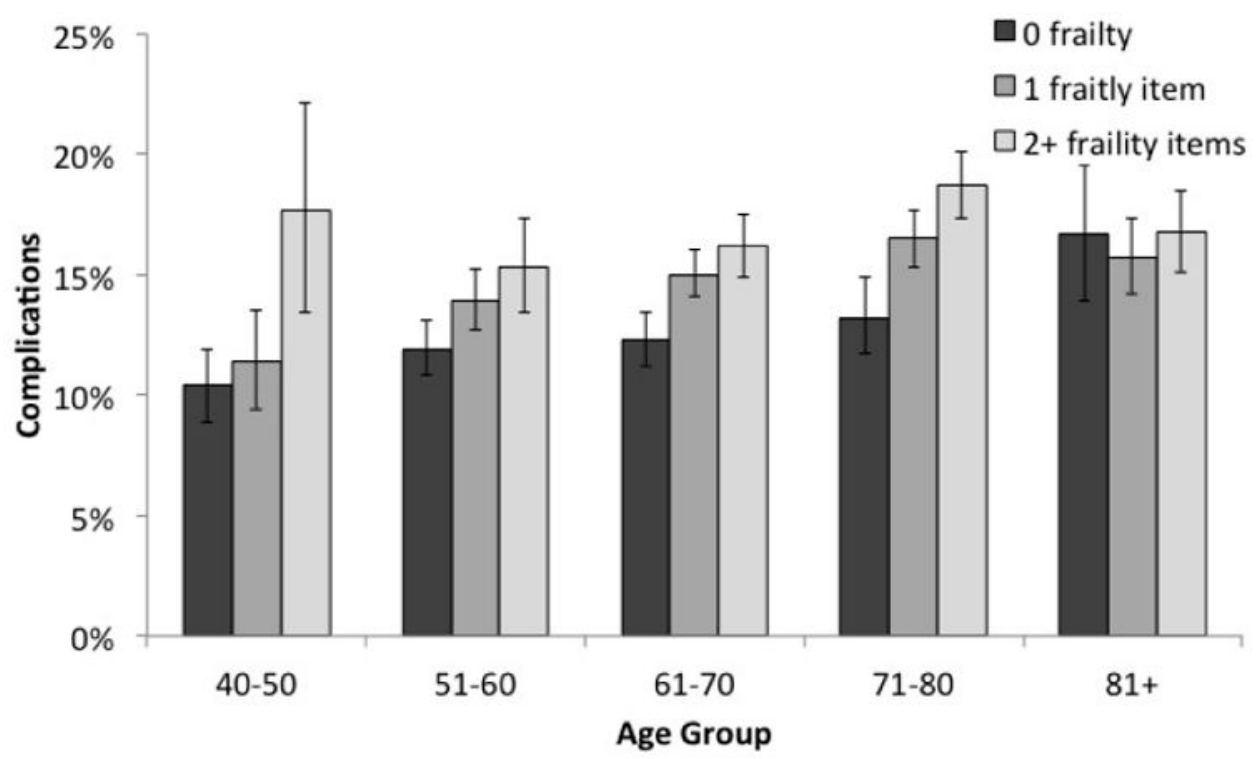

Figure 3.

Overall complications of urologic procedures according to age and NSQIP Frailty Index. 


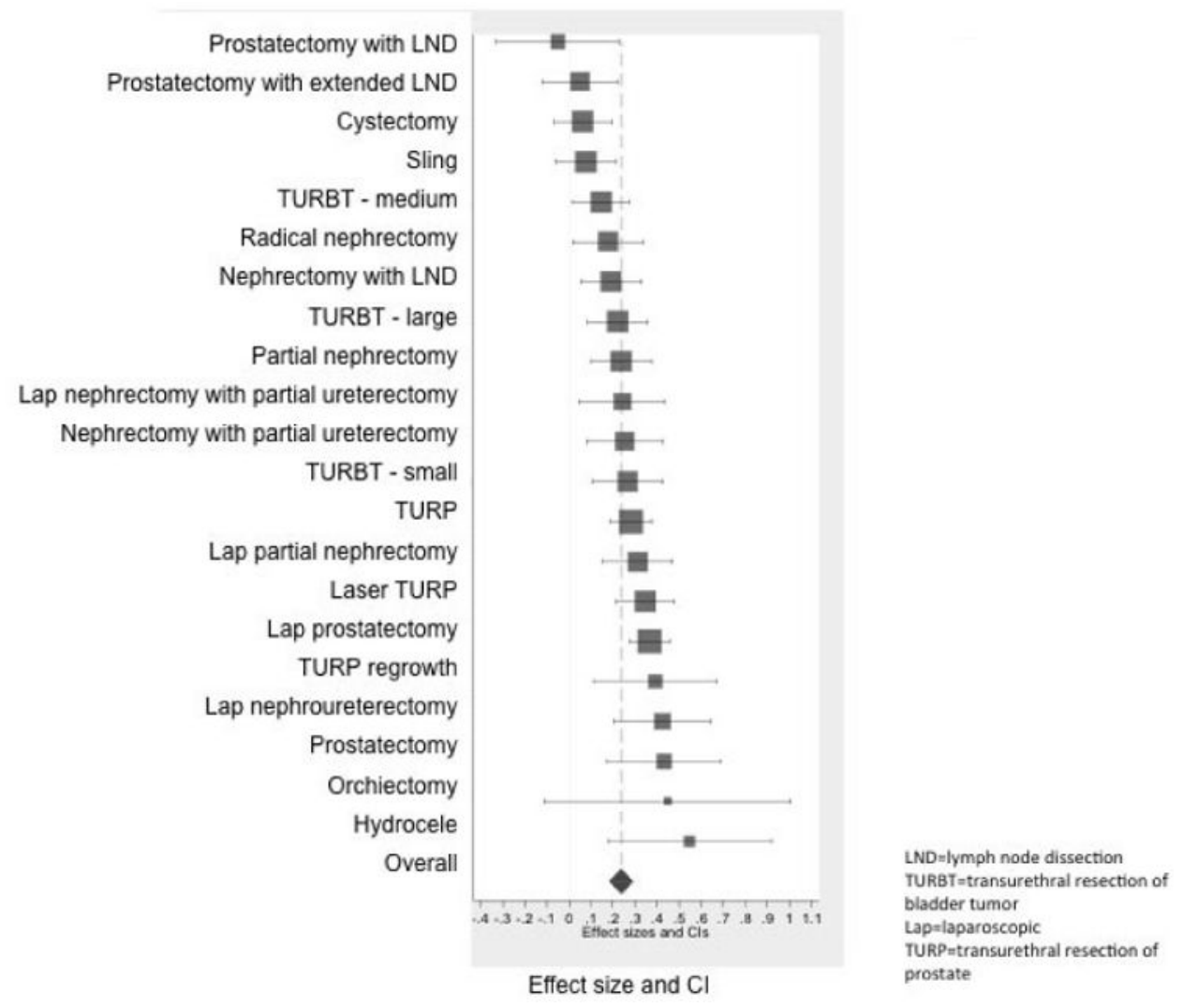

Figure 4.

Impact (log odds) of increasing NSQIP FI on complications associated with common urologic procedures. Data represent a multivariable logistic regression model adjusted for year, age, race, type of anesthesia, and smoking status. 
Table 1

Characteristics of patients undergoing common urologic procedures $(\mathrm{N}=95,108)$

\begin{tabular}{|c|c|c|}
\hline & Frequency & Percent \\
\hline \multicolumn{3}{|l|}{ Year } \\
\hline 2007 & 1,142 & 1.2 \\
\hline 2008 & 4,191 & 4.4 \\
\hline 2009 & 7,607 & 8.0 \\
\hline 2010 & 9,903 & 10.4 \\
\hline 2011 & 19,088 & 20.1 \\
\hline 2012 & 24,512 & 25.8 \\
\hline 2013 & 28,665 & 30.1 \\
\hline \multicolumn{3}{|l|}{ Age } \\
\hline $40-50$ & 9,316 & 10.2 \\
\hline $51-60$ & 20,242 & 22.2 \\
\hline $61-70$ & 30,581 & 33.5 \\
\hline $71-80$ & 20,702 & 22.7 \\
\hline $81+$ & 10,555 & 11.6 \\
\hline \multicolumn{3}{|l|}{ Race } \\
\hline White & 70,313 & 89.4 \\
\hline Black & 5,775 & 7.3 \\
\hline Other & 2,554 & 3.3 \\
\hline \multicolumn{3}{|l|}{ Anesthesia } \\
\hline General & 84,615 & 89.0 \\
\hline MAC & 2,313 & 2.4 \\
\hline Other & 8,127 & 8.6 \\
\hline \multicolumn{3}{|l|}{ Smoking status } \\
\hline Smoker & 14,537 & 15.3 \\
\hline Non-smoker & 80,571 & 84.7 \\
\hline \multicolumn{3}{|c|}{ Recent weight loss } \\
\hline No & 94,212 & 99.1 \\
\hline Yes & 895 & 0.9 \\
\hline \multicolumn{3}{|c|}{ NSQIP * railty Index } \\
\hline $\mathbf{0}$ & 37,596 & 39.5 \\
\hline .09 & 37,034 & 38.9 \\
\hline .18 & 16,147 & 17.0 \\
\hline .27 & 3,394 & 3.6 \\
\hline .36 & 747 & 0.8 \\
\hline $.45+$ & 190 & 0.2 \\
\hline
\end{tabular}

BJU Int. Author manuscript; available in PMC 2016 May 01. 


\section{Table 2}

Frequencies of individual complications among 95,108 urologic procedures 2007-2013.

\begin{tabular}{|c|c|c|}
\hline Complication & Number & Percent \\
\hline \multicolumn{3}{|c|}{ Minor complications } \\
\hline Blood transfusion & 4380 & 4.6 \\
\hline UTI & 2993 & 3.1 \\
\hline Pneumonia & 554 & 0.6 \\
\hline Superficial wound infection & 593 & 0.6 \\
\hline \multicolumn{3}{|c|}{ Major complications } \\
\hline Readmission & 1042 & 6.2 \\
\hline Return to OR & 1838 & 1.9 \\
\hline Sepsis & 801 & 0.8 \\
\hline DVT & 488 & 0.5 \\
\hline CVA & 440 & 0.5 \\
\hline Reintubation & 440 & 0.5 \\
\hline Death & 441 & 0.5 \\
\hline Renal failure & 349 & 0.4 \\
\hline MI & 288 & 0.3 \\
\hline PE & 315 & 0.3 \\
\hline Septic shock & 272 & 0.3 \\
\hline Dehiscence & 214 & 0.2 \\
\hline Cardiac Arrest & 147 & 0.2 \\
\hline Deep wound infection & 117 & 0.1 \\
\hline Coma & $*$ & 0.0 \\
\hline Overall & 11081 & 11.7 \\
\hline
\end{tabular}

* number too small to report based on NSQIP criteria 


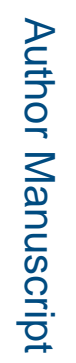

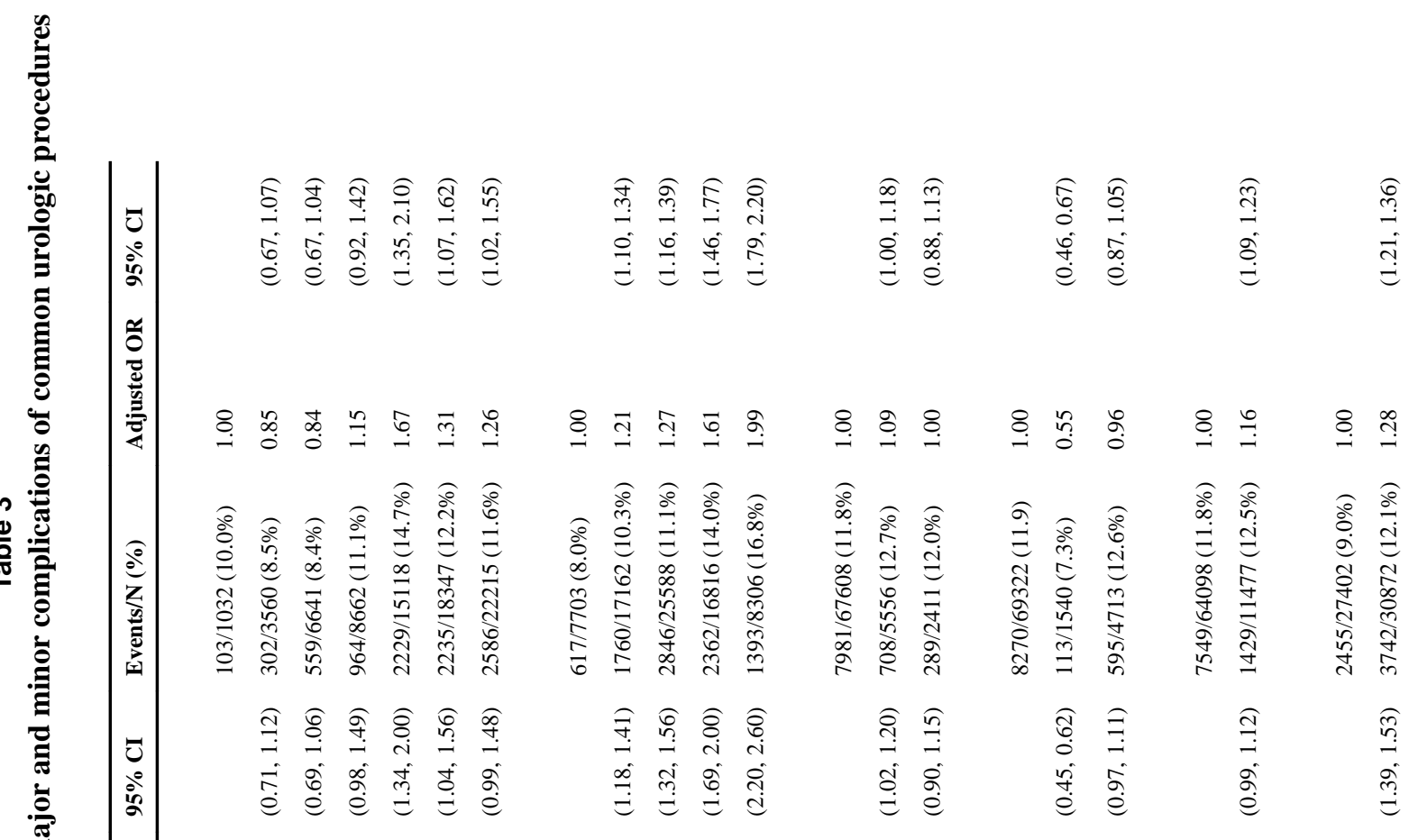

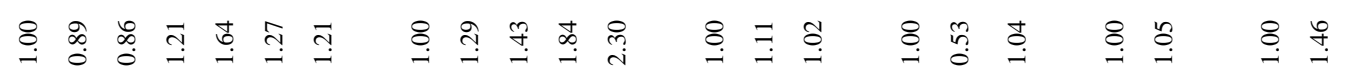

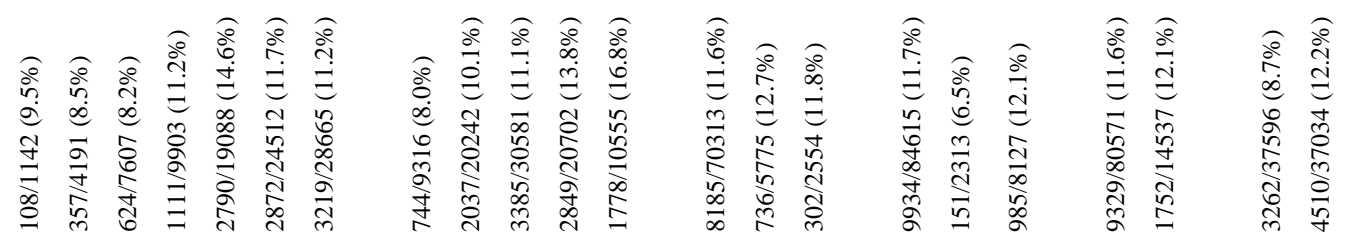

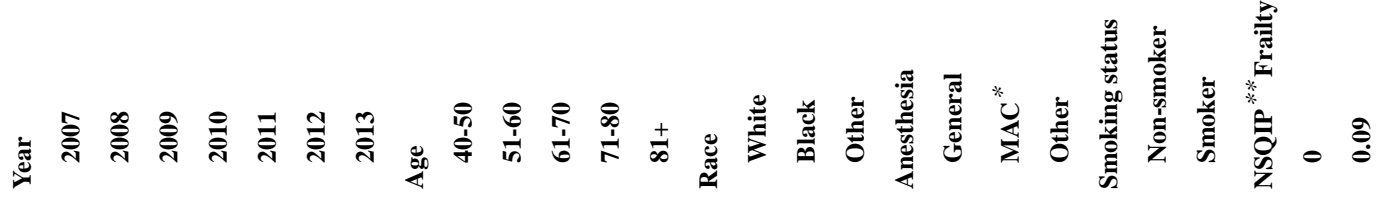
BJU Int. Author manuscript; available in PMC 2016 May 01. 
Suskind et al.

Page 16

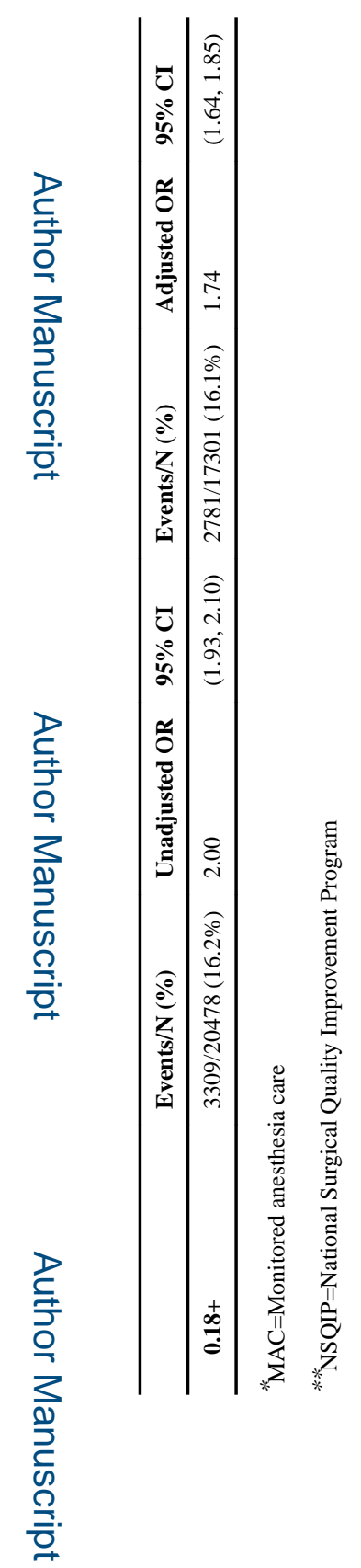

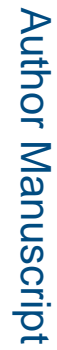

BJU Int. Author manuscript; available in PMC 2016 May 01. 